\title{
Autotransfusão de coleta pré-operatória em cirurgia cardíaca
}

Fábio B. JATENE ${ }^{\star}$, Pablo M. A. POMERANTZEFF*, Ana Cristina MONTEIRO**, Rafael ESTEBES**, Maria Cristina SILVA ${ }^{\star \star}$, Milton BECHARA ${ }^{\star \star}$, Yoshitaka OKUMURA***, Geraldo VERGINELLI* ${ }^{\star}$, Adib D. JATENE*

JATENE, F. B.; POMERANTZEFF, P. M. A.; MONTEIRO, A. C.; ESTEBeS, R.; SILVA, M. C.; BeChARA, M.; OKUMURA, Y.; VERGINELLI, G.; JATENE, A. D. - Autotransfusão de coleta pré-operatória em cirurgia cardíaca. Rev. Bras. Cir. Cardiovasc., 3(1):29-35, 1988.

RESUMO: A autotransfusão (AT) que utiliza a reposição de sangue autógeno constitui uma alternativa para a redução das complicaçōes da transfusão de sangue homólogo, já que, em cirurgias eletivas, existe a possibilidade da coleta de sangue no periodo pré-operatório. Foi realizado um estudo comparativo prospectivo para tentar estabelecer os parâmetros ideais da AT pré-operatória, referentes ao volume, intervalo de tempo prévio à cirurgia e benefício aos pacientes, entre outros. Foram considerados, para o estudo, 96 pacientes consecutivos a serem submetidos a revascularização miocárdica. As cirurgias foram eletivas e os pacientes não apresentavam hipoproteinemia, anemia, infecções, operações cardiacàs prévias, outras cirurgias recentes, ou, entăo, mais de 70 anos de idade. Foram divididos em 4 grupos; Grupo I: sem AT pré (controle, 41 pacientes); Grupo II: Coleta de 500 a $600 \mathrm{ml}$, sendo Grupo II-A até 7 dias antes da operação ( 35 pacientes) e Grupo II B de 8 a 15 dias antes (14 pacientes) e Grupo III com coleta realizada acima de 30 dias da operaçâo, com reinfusão e nova coleta a cada 15 dias (6 pacientes). A idade média e o hematócrito foram comparáveis, nos 4 grupos. Em alguns pacientes, utilizou-se, também, a AT no pós-operatório. Os resultados revelaram que $63 \%$ do Grupo I, $26 \%$ no Grupo II-A, $43 \%$ no Grupo II-B e $67 \%$ no Grupo III receberam sangue homólogo. A média de sangue autógeno recebido foi de 534 $\mathrm{ml} /$ paciente no Grupo II-A, $539 \mathrm{ml} /$ paciente no Grupo II-B e $908 \mathrm{ml} /$ paciente no Grupo III. O hematócrito, no pós-operatório, foi comparável nos 4 grupos, por ocasião da alta. Não houve óbitos. Os autores concluem que um menor número de pacientes do Grupo II-A recebeu sangue homólogo (ES $p=0,008)$ e não houve diferença estatística (ES) entre os grupos II-A, II-B e III, com relação ao volume de sangue homólogo recebido. Além disto, não houve aumento de complicações, particularmente sangramento, em nenhum dos grupos em relação ao grupo controle.

DESCRITORES: autotransfusāo, cirurgia cardiaca; sangue, reaproveitamento; circulação extracorpórea, reaproveitamento de sangue.

\section{INTRODUÇĀO}

A autotransfusão (AT) é um método que utiliza sangue autógeno na reposição volêmica, em uma série de situações quase sempre cirúrgicas.
Dentre as suas modalidades, destacam-se a AT de coleta pré-operatória, a intra, a per, a pós-operatória, $\mathrm{ou}$, ainda, em casos de hemotórax traumático ${ }^{2}, 6,11,12$. Estas modalidades podem utilizar o sangue total na forma em que é colhido, ou, então, os componentes sangüi-

\footnotetext{
Trabalho realizado no Instituto do Coração do Hospital das Clínicas da Faculdade de Medicina da Universidade de Sâo Paulo e no Serviço de Hemoterapia do Hospital das Clínicas da Faculdade de Medicina da Universidade de São Paulo. Săo Paulo, SP, Brasil.

Apresentado ao 15: Congresso Nacional de Cirurgia Cardiaca. Rio de Janeiro, 8 e 9 de abril, 1988.

- Do Instituto do Coraçāo do Hospital das Clínicas da Faculdade de Medicina da Universidade de São Paulo.

*. Da Faculdade de Medicina e do Hospital das Clínicas da Universidade de Săo Paulo.

... Do Serviço de Hemoterapia do Hospital das Clínicas da Faculdade de Medicina da Universidade de São Paulo.

Endereço para separatas: Fábio Jatene. Av. Dr. Eneas Carvalho de Aguiar, 44. Divisão Cirúrgica, 05403. Săo Paulo, SP, Brasil.
} 
JATENE, F. B.; POMERANTZEFF, P. M. A.; MONTEIRO, A. C.; ESTEBES, R.; SiLVA, M. C.; BeCHARA, M.; OKUMURA, Y.; VERGINELLI, G.; JATENE, A. D. - Autotransfusão de coleta pré-operatória em cirurgia cardiaca. Rev. Bras. Cir. Cardiovasc., 3(1): 29-35, 1988.

neos, geralmente concentrados de hemácias, após processamento e fracionamento em dispositivos próprios ${ }^{9}$.

Embora constituindo uma alternativa para a redução das transfusões sangüíneas homólogas, foi somente nos últimos anos que a AT ganhou maior impulso e aceitaçāo. Isto se deveu, sobretudo, ao aumento da incidência da síndrome da imunodeficiência adquirida (AIDS), que se somou às complicaçōes transfusionais anteriormente conhecidas ${ }^{3}, 8,10,17$.

Em cirurgia cardíaca, a AT já vem sendo rotineiramente empregada em inúmeros Centros nas suas várias modalidades, inclusive em nosso meio ${ }^{7}, 16,19,20$. Há vários anos vem sendo utilizada no Hospital das Clínicas e no Instituto do Coração, tendo sido proposto, inclusive, um dispositivo para sua realização ${ }^{12}$.

Este trabalho visa à avaliação da utilizaçăo e padronização da modalidade pré-operatória em cirurgia cardiaca.

\section{CASUISTICA E MÉTODOS}

A partir de março de 1987, foram considerados, para o estudo, 96 pacientes consecutivos submetidos, eletivamente, a cirurgia para revascularização do miocárdio. Foram excluídos pacientes com hipoproteinemia (proteínas totais $<6 \mathrm{~g} / 100 \mathrm{ml}$ ), anemia (hematócrito $<40 \%$ ), infecçōes de qualquer etiologia, operaçōes cardíacas prévias outras cirurgias recentes, ou idade superior a 70 anos. Estes pacientes foram distribuídos em 4 grupos a saber: Grupo I: pacientes em que não foi realizada coleta de sangue autógeno no pré-operatório (controle); Grupo II: onde foi colhida uma unidade de sangue no período do pré-operatório, sendo Grupo II-A até 7 dias antes da cirurgia e Grupo II-B 8 a 15 dias antes da cirurgia; Grupo III: coleta realizada 30 dias antes da operação, com reinfusões e novas coletas a cada 15 dias.

O número de pacientes, bem como a idade e o sexo, estão representados na Tabela 1.

\section{TABELA 1}

\section{NÚMERO DE PACIENTES IDADE E SEXO POR GRUPO}

\begin{tabular}{lccc}
\hline Grupos & Pacientes & Idade (anos) & Sexo masculino \\
I & 41 & $36-65(54,1 \pm 7,3)$ & $30(73 \%)$ \\
II-A & 35 & $39-65(54,6 \pm 7,4)$ & $30(86 \%)$ \\
II-B & 14 & $42-64(53,2 \pm 7,8)$ & $13(93 \%)$ \\
III & 6 & $40-69(61,5 \pm 11,5)$ & $6(100 \%)$ \\
\hline
\end{tabular}

As médias das pontes realizadas por pacientes, bem como os valores do hematócrito e das plaquetas, encontram-se na Tabela 2. O coagulograma foi normal, em todos os pacientes. Todos estes valores referem-se a exames laboratoriais realizados após a coleta do sangue autógeno.

TABELA 2

NÚMERO DE PONTES REALIZADAS E VALORES DO HEMATOCAITO E DAS PLAQUETAS EM CADA UM DOS 8 GRUPOS

\begin{tabular}{cccc}
\hline Grupos & Pontes (média) & Hematocrito & Plaquetas (média) \\
I & 2,7 & $45,4 \pm 3,5$ & 215.000 \\
II-A & 2,8 & $42,4 \pm 4,2$ & 210.000 \\
II-B & 2,8 & $43,8 \pm 3,6$ & 210.000 \\
III & 3,1 & $40,8 \pm 3,6$ & 196.000 \\
\hline
\end{tabular}

A retirada e a reinfusão do sangue, em todos os casos, seguiram as técnicas estabelecidas pelo Banco do Sangue do Hospital das Clínicas da FMUSP. Foram utilizadas bolsas plásticas*, contendo CPDA $(63 \mathrm{ml})$ para a coleta, e o armazenamento médio de 500 ml, correspondente a uma unidade de sangue.

A coleta de sangue, nos Grupos II-A e II-B, foi realizada na enfermaria, após a internaçāo do paciente, com retirada de uma unidade. Os pacientes do Grupo III foram convocados ambulatoriamente, para a retirada de 1 unidade, com retorno em 15 dias, para reinfusão desse sangue e nova coleta de 2 unidades. Quinze dias após, foram realizadas internação, nova reinfusão, coleta de 2 unidades e o paciente, liberado para cirurgia.

A autotransfusão pós-operatória (AT-PO) foi realizada em 19 pacientes, com utilização de recipiente específico $^{* \star}$, quando havia sangramento aumentado no pósoperatório imediato.

\section{RESULTADOS}

No Grupo I, 26 pacientes (63\%) receberam sangue homólogo, numa quantidade média de $448 \mathrm{ml} /$ paciente. Em $10(24 \%)$ foi utilizada AT-PO com média de 113 $\mathrm{ml} /$ paciente; neste grupo, foram observados hematócrito médio de $34,2 \pm 4,6 \%$, coagulograma sem alteraçōes significativas e contagem de plaquetas de $114.500 / \mathrm{mm}^{3}$, em média, no pós-operatório imediato.

No Grupo II-A, a média de sangue autógeno recebida foi $534 \mathrm{ml} /$ paciente, sendo que, neste grupo, 9 (26\%) pacientes também receberam sangue homólogo numa quantidade média de $193 \mathrm{ml} /$ paciente. Em 5 (14\%) pacientes, foi utilizada AT-PO, com $70 \mathrm{ml} /$ paciente. O hematócrito médio, nos pós-operatório imediato, foi 32,2 $\pm 4,7 \%$, apresentando, também, coagulograma normal e a contagem média de plaquetas de $128.800 / \mathrm{mm}^{3}$.

\footnotetext{
- Fenwal Laboratories.

** Bomba de autotransfusāo (BAT) - Macchi Engenharia Biomédica.
} 
JATENE, F. B.; POMERANTZEFF, P. M. A.; MONTEIRO, A. C.; ESTEBES, R.; SILVA, M. C.; BECHARA, M.; OKUMURA, Y; VERGINELLI, G.; JATENE, A. D. - Autotransfusão de coleta pré-operatória em cirurgia cardiaca. Rev. Bras. Cir. Cardiovasc., 3(1): 29-35, 1988.

No Grupo II-B, a média de sangue autógeno recebida foi $539 \mathrm{ml} /$ paciente, sendo que $6(43 \%)$ receberam sangue homólogo numa quantidade média de $439 \mathrm{ml}$. Em $3(21 \%)$ pacientes, foi usada a AT-PO com reinfusão média de $109 \mathrm{ml} /$ paciente. O hematócrito médio foi de $32,8 \pm 3,3 \%$; o coagulograma manteve-se nos limites da normalidade e a contagem de plaquetas em média foi de $115.000 / \mathrm{mm}^{3}$.

No Grupo III, a média de sangue autógeno recebida foi de $908 \mathrm{ml} /$ paciente, sendo que $4(67 \%)$ receberam sangue homólogo em quantidade média de $390 \mathrm{ml} / \mathrm{pa}$ ciente e, na AT-PO foram reinfundidos, em média, 58 $\mathrm{ml} /$ paciente. O hematócrito médio observado no pós-operatório imediato foi de $31,2 \pm 3,2 \%$; o coagulograma manteve-se normal e a contagem média de plaquetas foi de $106.000 / \mathrm{mm}^{3}$.

$\mathrm{Na}$ Tabela 3, estāo representadas as várias modalidades de transfusão recebida por cada grupo de pacientes, bem como o total de sangue recebido, em média, por paciente.

\section{TABELA 3}

QUANTIDADES RECEBIDASEM MÉDIA POR PACIENTE (EM ML) EM FUNÇĀO DA MODALIDADE DE TRANSFUSĀO, COM AS RESPECTIVAS PORCENTAGENS PROPORCIONAIS, POR CADA GRUPO

\begin{tabular}{lccrr}
\hline Grupos & SH & AT-Pré & \multicolumn{1}{c}{ AT-POS } & \multicolumn{1}{c}{ TOTAL } \\
I & $448(80 \%)$ & - & $113(20 \%)$ & $561 \mathrm{ml}$ \\
II-A & $193(24 \%)$ & $534(67 \%)$ & $70(9 \%)$ & $797 \mathrm{ml}$ \\
II-B & $439(40 \%)$ & $539(50 \%)$ & $109(10 \%)$ & $1087 \mathrm{ml}$ \\
III & $390(28 \%)$ & $908(66 \%)$ & $58(6 \%)$ & $1356 \mathrm{ml}$ \\
\hline
\end{tabular}

$\mathrm{SH}=$ sangue homólogo

AT $=$ autotransfusāo

A quantidade média de sangramento pós-operatório foi de $492,1 \pm 366,4 \mathrm{ml}$ no Grupo l; foi de $452,7 \pm$ $385,5 \mathrm{ml}$ no Grupo II-A e $545,0 \pm 402,7 \mathrm{ml}$ no Grupo II-B; finalmente, foi de $443,3 \pm 108,7 \mathrm{ml}$ no Grupo III.

Não houve óbito em nenhum dos 4 grupos.

Os resultados obidos foram estatisticamente avaliados e as variáveis, como o sexo e a idade, não tiveram influência nos resultados e nas análises posteriores.

A média dos volumes de sangramento pós-operatório (Gráfico 1) foi avaliada e não houve diferença estatisticamente significativa entre os 4 grupos.

Com relação à transfusão de sangue homólogo (Gráfico 2), houve, no Grupo II-A, menor porcentagem de pacientes que receberam sangue homólogo (SH), em relação aos outros grupos $(p=0,008)$.

No Gráfico 3 , está representado o volume total de sangue transfundido em relação à porcentagem de $\mathrm{SH}$. $\mathrm{Na}$ parte superior do gráfico, observa-se a média dos volumes totais de sangue recebido pelos pacientes $(\mathrm{SH}$, BAT e AT pré-op.); Grupo I recebeu menor quantidade total de sangue, quando comparado aos outros grupos $(p=0,0025)$. A parte inferior mostra a média das porcen- tagens de sangue homólogo em relação ao volume total recebido e não se observou diferença estatisticamente significativa entre os grupos.

\section{GRÁFICO}

MÉDIA DOS VOLUMES DE SANGRAMENTO NO PÓS-OPERATÓRIO IMEDIATO, SEM DIFERENCCA (ESTATISTICAMENTE SIGNIFICATIVA $P=0,008$ )

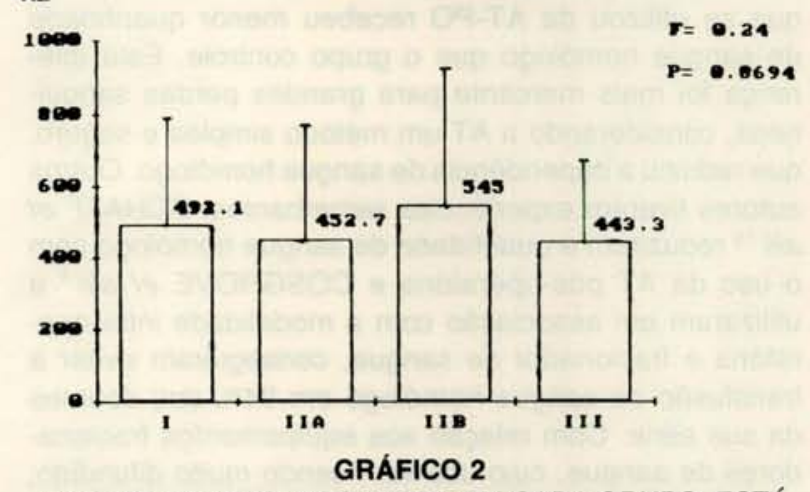

NAS COLUNAS DE ESQUERDA EM CADA GRUPO, ESTA A PORCENTAGEM DE PACIENTES QUE NÄO RECEBERAM $S H, E, A$ DIREITA, DOS QUE RECEBERAM. NO GRUPO $\|-A$, UMA MENOR PORCENTAGEM RECEBEU SH

× (ESTATISTICAMENTE SIGNIFICATIVA $P=0,008$ )

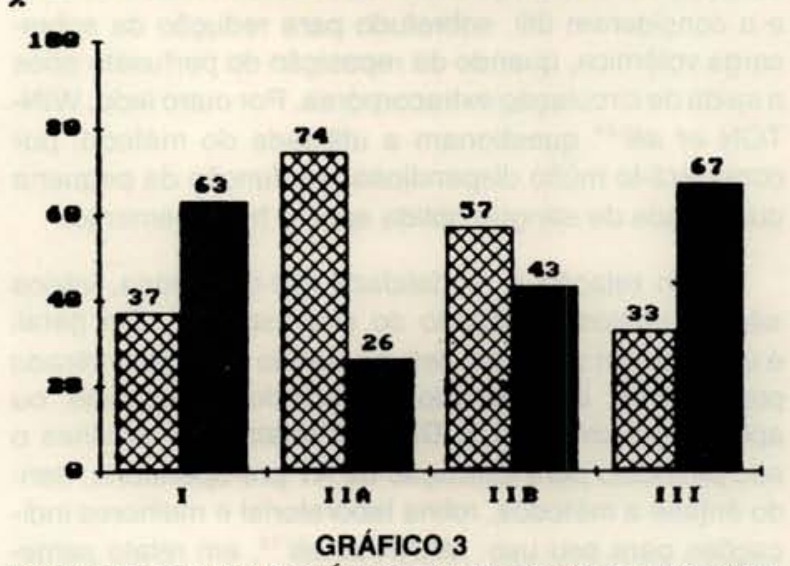

NA PARTE DE CIMA ESTAO TOTAL DE SANGUE RECEBIDO PELOS PACIENTES EM CADA GRUPOE, ABAIXO, A MÉDIA DAS PORCENTAGENS DE SH EM RELAÇAOAO VOLUME TOTAL DE SANGUE TRANSFUNDIDO

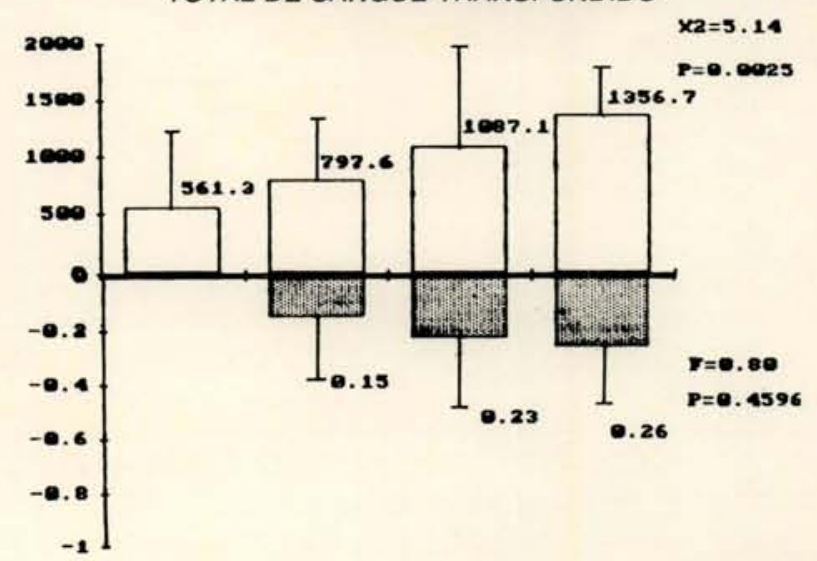


JATENE, F. B.; POMERANTZEFF, P. M. A.; MONTEIRO, A. C.; ESTEBES, R.; SILVA, M. C.; BeChARA, M.; OKUMURA, Y.; VERGINELLI, G.; JATENE, A. D. - Autotransfusão de coleta pré-operatória em cirurgia cardiaca. Rev. Bras. Cir. Cardiovasc., 3(1): 29-35, 1988.

\section{DISCUSSÃO}

Embora a autotransfusāo seja conhecida há mais de um século e meio, foi somente nos últimos anos que passou a ser mais empregada. Em cirurgia cardiaca, inúmeros trabalhos ressaltaram sua utilidade, sobretudo na modalidade pós-operatória. JOHNSON et alii ${ }^{13}$ a utilizaram em estudo comparativo e concluíram que o grupo que se utilizou da AT-PO recebeu menor quantidade de sangue homólogo que o grupo controle. Esta diferença foi mais marcante para grandes perdas sangüineas, considerando a AT um método simples e seguro, que reduziu a dependência de sangue homólogo. Outros autores tiveram experiências semelhantes. SCHAFF et alii ${ }^{19}$ reduziram a quantidade de sangue homólogo com o uso da AT pós-operatória e COSGROVE et alii ${ }^{4}$ a utilizaram em associação com a modalidade intra-operatória e fracionador de sangue, conseguiram evitar a transfusão de sangue homólogo em $94 \%$ dos doentes da sua série. Com relaçāo aos equipamentos fracionadores de sangue, cujo uso vem sendo muito difundido, existem algumas controvérsias. ANSELL et alii ${ }^{1}$, que utilizaram técnica com isótopos para estudar os efeitos do fracionamento sangüíneo sobre a vida média das hemácias, concluíram que o procedimento é seguro e confiável. GALANTIER et alii ${ }^{7}$, em nosso meio, a utilizam e a consideram útil, sobretudo para redução da sobrecarga volêmica, quando da reposição do perfusato após a saída de circulação extracorpórea. Por outro lado, WINTON et alii ${ }^{24}$ questionam a utilidade do método, por considerá-lo muito dispendioso, em função da pequena quantidade de sangue obtida após o fracionamento.

Com relação à modalidade pré-operatória, vários são os relatos a respeito do seu uso ${ }^{21}, 23$. Em geral, é utilizada em cirurgias eletivas, sendo o sangue retirado previamente, armazenado e infundido por ocasião, ou após, 0 ato cirúrgico ${ }^{18}$. DAVIS ${ }^{5}$ relata com detalhes o seu protocolo para utilização da AT pré-operatória, dando ênfase a métodos, rotina laboratorial e melhores indicações para seu uso. MANN et alii ${ }^{15}$, em relato semeIhante, em pacientes considerados de alto risco, concluí- ram que esta população não apresentou maiores complicaçōes na retirada do sangue para AT pré-operatória, podendo ser considerada para tal procedimento. LOVE et alii ${ }^{14}$, que a utilizaram em pacientes a serem submetidos a cirurgia cardíaca, consideraram o método simples e seguro. Além disto, chamam a atençāo para os benefícios de tal procedimento, sobretudo com relação à reduçāo da transfusāo de sangue homólogo e dos riscos das doenças inerentes à mesma.

Com relação ao presente trabalho, desde o início, alguns objetivos orientaram sua realização. Fundamentalmente, se buscou avaliar a utilidade e as melhores formas de realizar a AT pré-operatória. Procurou-se verificar se, com a utilização da AT pré-operatória, houve redução do volume total de $\mathrm{SH}$ infundido aos pacientes, ou diminuição do número de pacientes que receberam $\mathrm{SH}$. Além disto, procurou-se esclarecer se houve aumento de complicaçōes, traduzidas por sangramento e, também, qual o comportamento do hematócrito, no pré e no pós-operatório. Por fim, qual seria, sob o ponto de vista técnico, a melhor maneira de se realizar a AT e, conseqüentemente, qual o grupo mais favorável, com relação ao total de sangue colhido e, também, com relação ao período de coleta prévia à cirurgia.

Após o término da análise, observou-se que não houve aumento de complicaçōes (sangramento) em nenhum dos grupos, em relação ao grupo controle. A avaliação do hematócrito revelou um comportamento semelhante, nos 4 grupos, tanto no pré como no pós-operatório. Os pacientes do Grupo I receberam um menor volume total de sangue, sem diferença nos demais, seguramente em função de não receberem AT pré-operatória. Observou-se, ainda, que, no Grupo II-A, menor quantidade porcentual de pacientes recebeu $\mathrm{SH}$, quando comparada aos outros grupos. Esta relação foi estatisticamente significativa $(p=0,008)$.

Finalmente, não houve diferença estatisticamente significativa entre os Grupos II-A, II-B e III, com relaçăo ao volume de $\mathrm{SH}$ recebido. 
JATENE, F. B.; POMERANTZEFF, P. M. A.; MONTEIRO, A. C.; ESTEBes, R.; SiLVA, M. C.; BeChARA, M.; OKUMURA, Y.; VERGINELLI, G.; JATENE, A. D. - Autotransfusão de coleta pré-operatória em cirurgia cardiaca: Rev. Bras. Cir. Cardiovasc. 3(1): 29-35, 1988.

RBCCV 44205-46

JATENE, F. B.; POMERANTZEFF, P. M. A.; MONTEIRO, A. C.; ESTEBES, R.; SILVA, M. C.; BECHARA, M.; OKUMURA, Y.; VERGINELLI, G.; JATENE, A. D. - Pré-operative blood sampling for autotransfusion during cardiac surgery. Rev. Bras. Cir. Cardiovasc., 3(1): 29-35, 1988.

ABSTRACT: Autotransfusion (AT) is an alternative to reduce the incidence of complications following homologous blood transfusion. The utilization of autogenous blood is feasible in elective surgical procedures (Pre-AT). A prospective comparative study was carried out in order to establish the ideal parameters for pre-AT, concerning volume, time interval prior to surgery and benefits to the patient, among others. Ninety-six consecutive patients candidates for myocardial revascularization, were analized in the present study. These patients did not present hypoproteinemia, anemia, infections or more than 70 years of age; cardiac reoperations were discarded. Four groups $(\mathrm{G})$ were considered:

GI - control group, without pre-AT (41 patients); GII - collection of 500 to $600 \mathrm{ml}$, comprizing the groups; GII-A - until 7 days before surgery (35 patients); GII-B - from 8 to 15 days before surgery (14 patients); GIII - collection of $1000 \mathrm{ml}$ more than 30 days before operation, with reinfusion and a new collection after 15 days ( 6 patients). Mean age and hemotocrit were comparable in the different groups. In a few patients postoperative AT was utilized. The results indicated that homologous blood transfusion were necessary in $63 \%$ of GI, $26 \%$ of GII-A, $43 \%$ of GII-B and $67 \%$ of GIII patients. The mean amount of autogenous blood reinfused was $534 \mathrm{ml} /$ patient in Gll-A, $539 \mathrm{ml} /$ patient in Gll-B, and $908 \mathrm{ml} /$ patient in GIII. The postoperative hematocrit at discharge from the hospital was comparable in the four groups. There were no deaths. A smaller number of patients in Gll-A received homologous blood $(p=0,008$ ) and there was no statistically significant difference in the groups II-A, II-B and III concerning the volume of homologous blood received. There was no higher incidence of complications, specially bleeding, in the different groups when compared with the control group.

DESCRIPTORS: autotransfusion, cardiac surgery; blood re-utilization; extracorporeal circulation, blood re-utilization.

\section{REFERÊNCIAS BIBLIOGRÁFICAS}

1 ANSELL, J.; PARRILLA, N.; KING, M.; FOURNIER, L.; SZYMANSKY, I.; DOHERTY, P.; SALM, T. V.; CUTLER, B. - Survival of autotransfused red blood cells recovered from the surgical field during cardiovascular operations. J. Thorac. Cardiovasc. Surg., 84(3): 387-391, 1982.

2 BELL, W. - The hematology of autotransfusion. Surgery, 84(2): 695-699, 1978.

3 COLLINS, J. A. - Problems associeted with the massive transfusion of stored blood. Surgery, 75(2): 274-284, 1974.

4 Cosgrove, D. M.; THURER, B. L.; LYTLE, B. W.; PETER, M.; LOOP, F. D. - Blood conservation during myocardial revascularization. An. Thorac. Surg., 28(2): 184-189, 1979.

5 DAVIS, R. - Banked autologous blood for elevective surgery. Anaesth. Intens, Care, 71(1): 42-44, 1979.

6 FLEMING, A. W.; GREEN, D. C.; RADCLIFFE, J. H.; FLEMING, E. W. - Developement of a practical autologous blood transfusion program. $A m$. Surg., 43(1): 794-801, 1977.

7 GALANTIER, M.; BUB, R. F. GHIOTO, J. L.; TRINDADE, R. B.; SILVEIRA, F. S.; HAMERSCHLAK, N.; SZTERLING, L.; GOMES, K. D.; FÉHER, J. - Reaproveitamento do sangue em cirurgia extracorpórea: utilização de processadora por fluxo descontínuo. Rev. Bras. Cir. Cardiovasc., 2(1): 70-74, 1987.
8 GRADY, G. F. \& BENNETTI, J. E. - Risk of postransfusion hepatitis in the United States. JAMA, 220(5): 692-701, 1972.

9 ISBISTER, J. P. \& DAVIS, R. - Should autologous blood transfusion be rediscovered? Anaesth. Intens. Care, 8(2): 168-171, 1980.

10 JAMES, S. E.; DODDS, R.; SMITH, M. A. - Avoiding AIDS with autologous transfusions. $B r$. Med. J., 290(1): 854, 1985.

11 JATENE; F. B.; AMUCHI, W.; BECHARA, M. J.; HAZOFF, R. G.; LANGER, B.; BIROLINI, D. - Autotransfusão em hemotórax. Rev. Hosp. Clin. Fac. Med. S. Paulo, 38(4): 155-159, 1983.

12 JATENTE, F. B.; BECHARA, M. J.; LANGER, B.; BIROLINI, D.; OLIVEIRA, M. R. - Autotransfusão intra-operatória: novo dispositivo para sua realização. Rev. Paul. Med., 99(3): 30-33, 1982.

13 JOHNSON, R. G.; ROSENKRANTZ, K. R.; PRESTON, R. A.; HOPKINS, C.; DAGGETT, W. - The efficacy of postoperative autotransfusion in patients undergoing cardiac operations. Ann. Thorac. Surg., 36(2): 173-179, 1983.

14 LOVE, T. R.; HENDREN, W. G.; O'KEEFE, D. D.; DAGGETT, W. M. - Transfusion of predonated autologous blood in elective cardiac surgery. Ann. Thorac. Surg., 43(5): 508-512, 1987. 
JATENE, F. B.; POMERANTZEFF, P. M. A.; MONTEIRO, A. C.; ESTEBES, R.; SILVA, M. C.; BECHARA, M.; OKUMURA, Y.; VERGINELLI, G.; JATENE, A. D. - Autotransfusāo de coleta pré-operatória em cirurgia cardiaca. Rev. Bras. Cir. Cardiovasc., 3(1): 29-35, 1988.

15 MANN, M.; SACKS, H. J.; GOLDFINGER, D. - Safety of autologous blood donation prior to elective surgery for a variety of potentially "high-risk" patients. Transfusion, 23(3): 229-232, 1983.

16 POMERANTZEFF, P. M. A.; CHAMONE, D. F.; COSSERMELLI, M.; MARCIAL, M. B.; BELOTTI, G.; PILEGGI, F.; BITTENCOURT, D.; VERGINELLI, G. - Autotransfusão em cirurgia cardiaca. Arq. Bras. Cardiol., 41(4): 247-254, 1983.

17 PRINCE, A. M.; SZMUNESS, W.; MILLIAN, S. J.; DAVID, D. S. - A serologic study of cytomegalovirus infections associated with blood transfusions. N. Engl. J. Med., 284(19): 1125-1131, 1971.

18 Rebulla, P.; GiOVANetTI, A. M.; PETRINI, G.; MEZZETTI, M.; SIRCHIA, G. - Autologous blood predeposit for elective surgery: a program for better use and conservation of blood. Surgery, 97(4): 463-465, 1985.

19 SCHAFF, H. V.; HAUER, J.; GARDNER, T. J.; DONAHOO, J. S.; WATKINS, J. L.; GOTT, V. L.; BRAWLEY, R. K. - Routine use autotransfusion following cardiac surgery. Ann. Thorac. Surg., 27(6): 493-499, 1979.

20 TECTOR, A. J.; DRESSLER, D. K.; GLASSNER-DAVIS, M. - A new method of autotransfusing blood drained after cardiac surgery. Ann. Thorac. Surg., 40(3): 305-307, 1985.

21 TOY, P. T. C. Y.; STRAUSS, R. G.; STEHLING, L. C.; SEARS, R.; PRICE, T. H.; ROSSI, E. C.; COLLINS, M. L.; CROWLEY, J. P.; EISENSTAEDT, R. S.; GOODNOUGH, L. T.; GREENWALT, T. J.; JOHNSTON, M. F. M.; KENNEDY, M. S.; LENES, B. A.; LUSHER, J. M.; MINTZ, P. D.; PATTEN, E. D.; SIMON, T. L.;WESTPHAL, R. G. - Predeposited autologous blood for elective surgery. $\quad$ N. Engl. J. Med., 316(9): 517-520, 1987.

22 UTLEY, J. R.; MOORES, W. Y.; STEPHENS, D. B. Blood conservation techiniques. Ann. Thorac. Surg., 31(5): 482-490, 1981.

23 YOMTOVIAM, R. - Increasing the use of predeposited autologous blood for transfusion. N. Engl. J. Med., 317(9): 569-570, 1987.

24 WINTON, T. L.; CHARRETTE, E. J. P.; SALERNO, T. A. - The cell saver during cardiac surgery: does it save? Ann. Thorac. Surg., 16(1): 379-381, 1973.

AGRADECIMENTOS: Às Sras. Rita Helena A. Cardoso e Silvana De Peron Galucci, do Setor de Estatística do Serviço de Informática Médica do Instituto do Coraçâo do HC-FMUSP, pela avaliação estatística do trabalho.

\section{Discussão}

\section{DR. IVAN CASAGRANDE Belo Horizonte, MG}

Em primeiro lugar, gostaria de agradecer à Comissão Organizadora, pela escolha de meu nome para co- mentar este trabalho e, posteriormente, colocar aqui que, realmente, o problema de transfusão de sangue tem sido abordado em vários aspectos. Ao Dr. Fábio Jatene, gostaria de parabenizá-lo, pois, há bastante tempo, trabalha nessa área, tentando reduzir o uso de sangue homólogo nas transfusões. Com relação a este trabalho de pré-depósito, cabem algumas observaçōes. Nós acreditamos que possa haver diferença em vários programas de pré-depósito, nāo só na cirurgia cardiaca, como também para outros tipos de cirurgia, como foi demonstrado pelo $\mathrm{Dr}$. Fábio e colaboradores. No presente trabalho, os grupos de colheita apresentam comportamento diferente, quanto ao sangramento e quanto ao aproveitamento de sangue, no pós-operatório. Assim, acredito que os programas de pré-depósito deveriam ter, talvez, uma diferenciação para os determinados tipos de cirurgia. O levantamento publicado da Associação Americana dos Bancos de Sangue demonstra que houve um acréscimo, de 1981 a 1985 , de $48 \%$ para $62 \%$, com o uso de autotransfusão. Nos Estados Unidos, $65 \%$ dos Serviços de cirurgia cardíaca aplicam, hoje, pelo menos algum tipo de autotransfusão e, em hospitais de acima de 200 leitos, o uso de autotransfusão duplicou. Muito obrigado.

\section{DR. ROBERTO GOMES DE CARVALHO Curitiba, $P R$}

Na Segunda Guerra Mundial, o surto de hepatite foi impressionante, atingindo cerca de 122 mil pessoas, das quais 650 morreram por sangue contaminado. Mas, apesar de todo o avanço nos últimos anos, existem consideráveis riscos, que estão associados à transfusão homóloga em cirurgia cardíaca, como: hepatite não-A e não-B com incidência de 6 a $10 \%$ por unidade transfundida, reaçōes transfusionais que persistem em vários casos e a AIDS, com incidência de $27 \%$ dos pacientes operados. Diga-se que esta alta taxa se deva aos melhores resultados cirúrgicos tardios, tempo suficiente para aflorar a doença. Analisando o trabalho do Dr. Fábio e colaboradores, o que mais nos chamou a atenção foi a ausência de um estudo estatístico. Mas as conclusōes dos autores coincidem com o teste aplicado, em que os grupos II-A e III mostraram melhores resultados. Gostariamos de fazer alguns comentários, que dividiremos em 3 partes: pré, trans e pós-operatório. No pré: sabemos que o sangue estocado por mais de 7 dias pode levar a alteraçóes, tais como: o $\mathrm{K}^{+}$se eleva para $22 \mathrm{mEq} / 1, \mathrm{p}$ ph diminui para 6,8 , existe diminuição do cálcio e do hematócrito, bem como aumento das taxas de amônia, levando à hiperamoniemia. Por isso, a importância para os doentes do Grupo III. Nos doentes do Grupo II-B (coleta de 8 a 15 dias), não tem tempo médio. Isto porque faz diferença se este tempo está mais perto de 8 dias, ou de 15 dias. Não houve relato sobre se a bolsa plástica era nacional, ou importada; existe possibilidade de contaminação por Pseudomonas e E. coli. Não foi citado qual o tipo de anticoagulante. Dependendo 
JATENE, F. B.; POMERANTZEFF, P. M. A.; MONTEIRO, A. C.; ESTEBES, R.; SILVA, M. C.; BECHARA, M.; OKUMURA, Y.; VERGINELLI, G.; JATENE, A. D. - Autotransfusão de coleta pré-operatória em cirurgia cardiaca. Rev. Bras. Cir. Cardiovasc., 3(1): 29-35, 1988.

do tipo, o sangue pode ser conservado por mais tempo. $O$ anticoagulante ACD conserva o sangue por 21 dias, mas o CPDA (citrato-fosfato-destrose adenina) conserva 0 sangue por 35 dias e o Adson, por 42 dias. Outro detalhe é a idade do paciente. Quanto mais jovem, meIhor a capacidade de regeneração medular. Este fato é importante nos doentes do Grupo II-A. Ainda no pré-operatório, a administração de sulfato ferroso $300 \mathrm{mg}$, e por dia é benéfica e a interrupção do ácido acetilsalicílico é necessária, para evitar aumento de sangramento no pós-operatório. Em relação ao trans-operatório, o mais importante é o tempo de circulaçāo extracorpórea, pois, se for acima de 120 minutos, está descrito que a transfusão homóloga é bem maior, mesmo nos doentes que tiveram seu sangue pré depositado, com diferença estatística. A simples abertura da pleura, com drenagem do hemitórax, requer maior quantidade de sangue homólogo. Dependendo do tipo de anestésico, este pode levar a uma maior vasodilatação, com necessidade de se usar expansor plasmático, ou, mesmo, sangue homólogo. Quanto ao pós-operatório, cerca de $43,7 \%$ dos pacientes, no presente trabalho, receberam sangue homólogo, e seria interessante saber se houve complicações, a curto e longo prazo (AIDS), desses doentes. A AT-pós, apesar de eficiente, pode, em alguns casos, levar à hipofibrinogeniemia, alterando os fatores de coagulação. Em relação aos resultados obtidos de que $56 \%$ (Grupo I), $28 \%$ (Grupo II-A), 50\% (Grupo II-B) e 60\% (Grupo III) receberam $\mathrm{SH}$, pergunto: como foram obtidos estes dados? Sabendo que, em pacientes graves (lesão tronco, tri-arterial, angina instável), a diminuição da hemoglobina pode levar à isquemia, mesmo assim os autores realizam a AT-pré? Sem dúvida alguma, é um trabalho de suma importância, em que os autores souberam muito bem abordar e dar relevância à magnitude do atual problema, não somente perante o doente, mas também à Sociedade.

\section{DR. DOMINGO BRAILE \\ São José do Rio Preto, SP}

É lógico que queremos parabenizar o Dr. Fábio, e este trabalho, que vem de longe e é formidável. Eu só queria dar uma alternativa, que temos usado e parece ser útil para todos. Ao final da cirurgia com circulação extracorpórea, geralmente se tem um volume de sangue que sobra no oxigenador e é difícil de ser reintroduzido; acho que nem todos podem possuir um cell saver. Os Bancos de Sangue dispōem de centrífugas, que podem centrifugar as bolsas de sangue. Nós temos retirado esse sangue e centrifugado na centrífuga do Banco. Isto demora 20 minutos, mais ou menos, e nós podemos ter um sangue sem líqüido, com hematócrito geralmente em torno de $80 \%$, ou $75 \%$. Espandimos isto um pouco e, hoje, todo o sangue aspirado do campo, oli, mesmo, da cardioplegia é mandado para o Banco de Sangue para ser levado e aproveitar as hemácias. Acho que todo o esforço deva ser feito para que a gente guarde as hemácias do paciente. Isto tem-se mostrado, realmente, muito útil.

\section{DR. FÁBIO JATENE (Encerrando)}

Vou procurar ser breve e, inicialmente, gostaria de agradecer ao Dr. Ivan Casagrande e ressaltar que este trabalho não é só nosso; outros médicos de nosso Serviço e outros profissionais têm colaborado; gostaria de ressaltar o Dr. Pablo Pomerantzeff, que é quem mais tem trabalhado junto conosco, nesse programa. Com relação às observações do Dr. Roberto Gomes de CarvaIho, só posso agradecer. Como ele mesmo ressaltou, grande parte das dúvidas que ele havia selecionado para o seu comentário foram elucidadas, durante a apresentação deste trabalho, sobretudo quanto à parte de estatística e quanto às observações da presença de anticoagulantes e, sobretudo, em relação à quantidade e tipos de anticoagulantes. No que se refere às suas duas observaçōes quanto ao tempo de circulação extracorpórea, ao número e às perspectivas das complicações que esses doentes apresentaram, eu gostaria de dizer que esses foram resultados levantados; entretanto, o que nós tínhamos era um grupo comparativo, quer dizer, um grupo controle em relaçăo aos outros grupos, e estes não eram os objetivos de nosso trabalho; quer dizer, não estávamos interessados em saber se isto provocou, ou resultou em complicaçōes adicionais. Efetivamente, estamos interessados em saber, no momento, outros dados e, como era um trabalho comparativo, achamos que, desde que tivesse havido importância entre o comportamento dos grupos, isto, efetivamente, não importava ser apresentado aqui. Quanto à última observaçāo do Dr. Roberto, sobre os pacientes graves, nós não temos utilizado o procedimento nestes doentes, mas sim naqueles submetidos, eletivamente, a cirurgia. Por fim, gostaria de agradecer ao Dr. Braile a gentileza de sua palavra e suas sugestôes tão pertinentes. Obrigado. 